\title{
Treating Refractory Mental Illness with Closed-Loop Brain Stimulation: Progress Towards a Patient-Specific Transdiagnostic Approach
}

\author{
Alik S. Widge ${ }^{\text {a,b,*}}$, Kristen K. Ellard ${ }^{\mathrm{a}}$, Angelique C. Paulk ${ }^{\mathrm{c}}$, Ishita Basu ${ }^{\mathrm{c}}$, Ali Yousefic, Samuel \\ Zorowitz ${ }^{\mathrm{a}}$, Anna Gilmour ${ }^{\mathrm{a}}$, Afsana Afzal ${ }^{\mathrm{a}}$, Thilo Deckersbach ${ }^{\mathrm{a}}$, Sydney S. Cash ${ }^{\mathrm{d}}$, Mark A. \\ Kramer ${ }^{\mathrm{e}}$, Uri T. Eden ${ }^{\mathrm{e}}$, Darin D. Dougherty ${ }^{\mathrm{a},{ }^{\dagger}}$ Emad N. Eskandar ${ }^{\mathrm{c}, \dagger}$
}

\begin{abstract}
Mental disorders are a leading cause of disability, morbidity, and mortality amongst civilian and military populations. Most available treatments have limited efficacy, particularly in disorders where symptoms vary over relatively short time scales. Targeted modulation of neural circuits, particularly through open-loop deep brain stimulation (DBS), showed initial promise but has failed in blinded clinical trials. We propose a new approach, based on targeting neural circuits linked to functional domains that cut across diagnoses. Through that framework, which includes measurement of patients using six psychophysical tasks, we seek to develop a closed-loop DBS system that corrects dysfunctional activity in brain circuits underlying those domains. We present convergent preliminary evidence from functional neuroimaging, invasive human electrophysiology, and human brain stimulation experiments suggesting that this approach is feasible. Using the Emotional Conflict Resolution (ECR) task as an example, we show that emotion-related networks can be identified and modulated in individual patients. Invasive and non-invasive methodologies both identify a network between prefrontal cortex, cingulate cortex, insula, and amygdala. Further, stimulation in cingulate and amygdala changes patients' performance in ways that are linked to the task's emotional content. We present preliminary statistical models that predict this change and allow us to track it at a single-trial level. As these diagnostic and modeling strategies are refined and embodied in an implantable device, they offer the prospect of a new approach to psychiatric treatment and its accompanying neuroscience.
\end{abstract}

Keywords: Deep brain stimulation ; psychiatric illness ; psychiatric diagnosis ; functional imaging ; mood disorders ; anxiety disorders ; electrophysiology ; modeling ; local field potential 
Abbreviations: DBS, deep brain stimulation; ECR, Emotional Conflict Resolution ; GAD, generalized anxiety disorder ; gMVR, generalized multivariate autoregressive ; MDD, major depressive disorder ; MVAR, multivariate auto-regressive modeling ; PPI, psychophysical interaction analysis ; PTSD, post-traumatic stress disorder ; SUD, substance use disorder 


\section{Introduction}

Mental disorders are the single largest cause of disability worldwide [1]. In the wake of recent military conflicts, they have become a major source of morbidity and mortality among warfighters and Veterans [2,3]. Disorders of concern in this population include post-traumatic stress disorder (PTSD), major depressive disorder (MDD), generalized anxiety disorder (GAD), substance use (SUD), and traumatic brain injury (TBI). Within those disorders, as with many other psychiatric diagnoses, there has been no change for decades in the overall mortality despite extensive research [4,5]. Psychotropic medications adjust neurotransmitter levels globally across the brain. This fails to address the mechanisms of mental illness. If depression were a monoamine deficit, or schizophrenia a syndrome of excess dopamine, antidepressants and antipsychotics would exceed their roughly 30-40\% efficacy [6-9]. The situation is worse for anxiety disorders, particularly PTSD and GAD [10,11]. Serotonin reuptake inhibitors (SRIs), the mainstay of evidence-based pharmacology for anxiety, have modest efficacy at best [12]. Benzodiazepines (BZDs) are much stronger anxiolytics, bringing immediate short-term relief. Unfortunately, that relief can become an avoidance behavior that reinforces and worsens maladaptive responses to anxiety. BZDs also have direct addictive potential and can be rapidly fatal in overdose. Psychotherapy, particularly exposure-based therapy, has substantially greater efficacy and is often considered the true first-line treatment $[13,14]$. Unfortunately, well-trained therapists are expensive and difficult for most patients to access

More recently, a new tool has emerged: targeted electro-magnetic brain stimulation. As mental illness became understood as a dysfunction of brain circuits, investigators have sought to reregulate those circuits $[15,16]$. We have known for decades that targeted stereotactic lesions can be remarkably effective even in refractory cases of depression and obsessive-compulsive disorder [17-23]. Stimulation may replicate the benefits of those lesions while also being reversible. Vagus nerve stimulation (VNS) and transcranial magnetic stimulation (TMS) are both approved for MDD [24-27]. The most promising approach, however, remains deep brain stimulation (DBS). Early open-label reports of DBS for MDD and OCD were encouraging, with high response rates even in treatment-resistant cases [28-30]. In randomized controlled trials, however, DBS for MDD failed to meet endpoints [31,32]. DBS for OCD is only available under a Humanitarian Device Exemption (HDE), because very few patients will ever qualify for surgery [33].

We attribute those failures to the neglect of a key fact: mental disorders are not static. Symptoms wax and wane across days or hours. In PTSD and anxiety disorders, they can flare and remit on the order of minutes. Standard DBS is open loop: it delivers a constant level of treatment, regardless of sleep-wake cycles, current symptom levels, or side effects. Adjustments occur only at clinical visits, weeks to months apart. This is a mis-match between the time course of the disease and the timing of clinical adjustments. One solution is closed loop stimulation, in which the device itself adjusts stimulation by inferring the patient's immediate clinical need from the brain's electrical activity [34,35]. Such approaches are clinically approved in epilepsy [36], and have shown promise in Parkinsons disease [37,38]. The challenge for psychiatric DBS is identifying the biomarkers - the neural signatures of mental illness and its fluctuating symptoms. Many investigators have sought these, often through neuro-imaging and electroencephalography (EEG). Despite decades of work, there is no known electrical signature of the symptoms of any mental illness. Many putative signatures do not replicate on subsequent testing [39-41]. 
In response to those challenges, we describe a new approach to psychiatric DBS, funded by the Defense Advanced Research Projects Agency (DARPA) as part of the Brain Research through Advancing Innovative Neurotechnologies (BRAIN) Initiative. The essence of the TRANSFORM DBS (Transdiagnostic Restoration of Affective Networks by Systematic, Function-Oriented Real-time Modeling and Deep Brain Stimulation) project is a transdiagnostic framework. We consider psychiatric disorders as embedded in a multi-axial space of "functional domains", similar to the National Institute of Mental Health's Research Domain Criteria (RDoC) framework [42]. We believe that these domains, being grounded in objectively measurable behavior, will have stronger and more replicable neural correlates compared to clinical psychiatric diagnoses [43]. We first overview the rationale for this domain-oriented framework, identify an initial set of functional domains, and link them to disorders of national military significance. We then present a series of preliminary experiments demonstrating that these domains can be measured both non-invasively and invasively in the awake, behaving human, that the electrical and behavioral measurements are amenable to mathematical modeling, and that those models may be used to develop brain stimulation that changes psychiatrically relevant behaviors. We conclude with discussion of the next steps to turn these concepts into a clinical device. All experiments described herein were approved by the Massachusetts General Hospital Institutional Review Board and were subject to second-level review by the Army's Human Subjects Research Protection Office (HRPO).

\section{Rationale and Coverage for the Transdiagnostic Framework}

Progress in the basic and clinical neurosciences has advanced our understanding of psychopathology. This has placed an increasing emphasis on dysfunction that cuts across domains of functioning (e.g., Negative Valence, Positive Valence, Cognitive Processes, Social Processes), as embodied in RDoC. That suggests the possibility of classifying an individual patient's mental illness based upon his/her specific patterns of dysfunction, rather than relying upon symptom clusters that show poor reliability [44]. A domain-oriented approach may address the high rates of comorbidity and heterogeneity found under the checklist-based system of the Diagnostic \& Statistical Manual of Mental Disorders (DSM) [45]. For TRANSFORM, we have adopted six initial domains: Fear Extinction, Reward Motivation, Emotion Regulation, Decision Making/Impulsivity, Cognitive Flexibility, and Learning/Memory. These overlap with RDoC constructs, but are more specifically tailored to the clinical disorders we hope to address. Each is impaired across disorders of military interest (Table 1), each has well-established and validated metrics, and each has been linked to specific brain areas and circuits.

The domain-oriented approach resolves two related problems: diagnostic overlap and heterogeneity within single diagnoses (Figure 1). First, patients with ostensibly different disorders may share strong common phenotypes. For example, PTSD patients frequently have deficits in Emotion Regulation, which is linked to brain connectivity between frontal regions and the amygdala [46-48]. That dysregulation is also commonly seen in MDD [49,50], and the two disorders are often comorbid [44,51,52]. PTSD and MDD also have a common deficit in Cognitive Flexibility: PTSD often involves perseveration on what a patient could/should have done in the moment, whereas MDD frequently has "stuck", ruminative thinking. The strong role of the prefrontal cortex (PFC) in mental flexibility [53-56] links MDD and PTSD to TBI (Figure 1A), which also shows perseverative behavior and often involves frontal injury. Thus, a DBS 
intervention that targeted Cognitive Flexibility could be applicable to multiple patient groups who, from a categorical perspective do not have the "same disease".

The other limitation of categorical diagnosis is heterogeneity. MDD is a prime example: with 9 diagnostic criteria, 5 of which must be present to confirm the diagnosis, there are 126 different clinical phenotypes, many of which have almost no symptoms in common. Individual patients with MDD may be profoundly emotionally labile (Emotion Regulation), flattened and anhedonic (Reward Motivation), or stuck in rigid, self-flagellating guilt (Cognitive Flexibility). DBS in any given brain circuit can likely only address one of those domains (Figure 1B), suggesting that applying it to the heterogeneous construct of "depression" is pre-destined to find only weak clinical signals. We believe this was the key weakness in recent DBS trials in MDD, and that our domain-focused approach can overcome it [43]. Put another way, patients with the same categorical diagnosis may have completely non-overlapping clinical or neurological phenotypes. The next two sections will give an example of such a situation and the ways in which the TRANSFORM approach can resolve it.
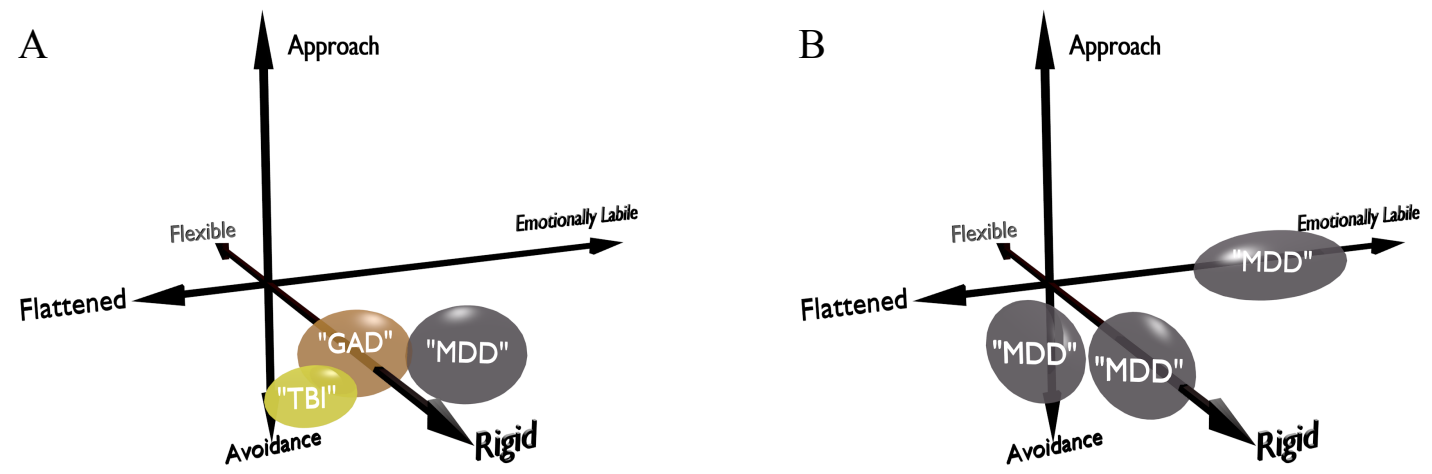

Figure 1: Schematic illustrations of transdiagnostic framework. (A), three patients with different DSM diagnoses may have the same phenotypic problem, e.g. cognitive rigidity.

(B), three patients with MDD (notionally the "same") may differ on the domains of cognitive flexibility, approach and avoidance to stimuli, and emotional lability. 
Table 1. Functional Domain-Based Assessment

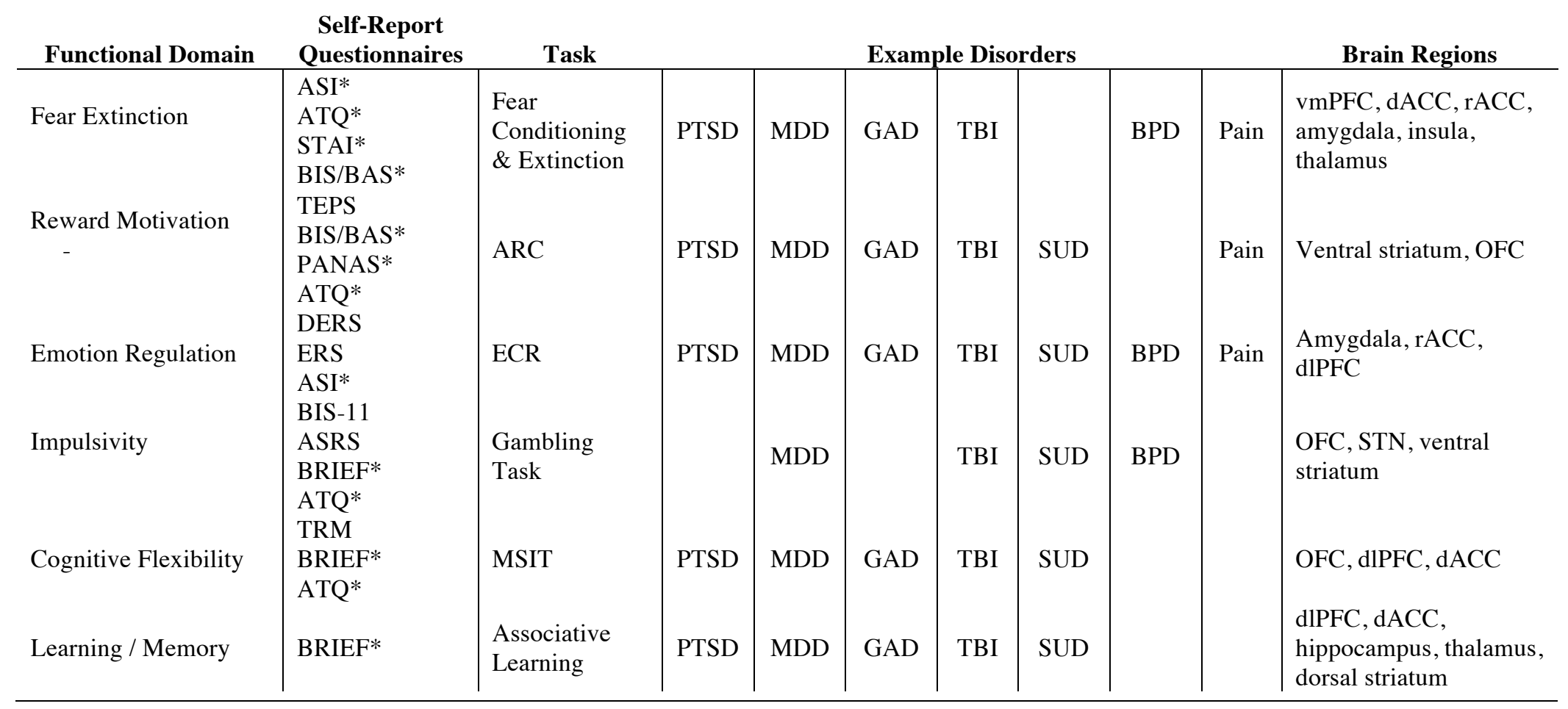

Table 1: TRANSFORM working map between domains, assessment instruments (self-report and psychophysical), DSM-5 disorders, and implicated brain regions. Questionnaires: ASI = Anxiety Sensitivity Index, ATQ = Adult Temperament Questionnaire, STAI = State-Trait Anxiety Inventory, BIS/BAS = Behavioral Inhibition/Behavioral Activation Scale, TEPS = Temporal Experience of Pleasure Scale, PANAS = Positive and Negative Affect Scale, DERS = Difficulties in Emotion Regulation Scale, ERS = Emotion Reactivity Scale, BIS-11 = Barratt Impulsivity Scale, ASRS = Adult ADHD Self-Rating Scale, BRIEF $=$ Brief Inventory of Executive Functioning, TRM = Trait Rumination Scale. Tasks: ARC = Aversion-Reward Conflict, ECR = Emotion Conflict Resolution, MSIT = Multiple Source Interference Task. Disorders: PTSD = Posttraumatic Stress Disorder, MDD = Major Depressive Disorder, GAD = Generalized Anxiety Disorder, TBI = Traumatic Brain Injury, SUD = Substance Use Disorder, BPD = Borderline Personality Disorder, Pain = Pain Disorders. Brain Regions: dlPFC $=$ dorsolateral prefrontal cortex, $\mathrm{dACC}=$ dorsal anterior cingulate, $\mathrm{OFC}=$ orbitofrontal cortex, $\mathrm{rACC}=$ rostral anterior cingulate, $\mathrm{STN}=$ subthalamic nucleus, $\mathrm{vmPFC}=$ ventromedial prefrontal cortex 
Table 1 also illustrates that each domain maps to a suite of self-report questionnaires and a validated psychophysical task. By comparing a patient's behavior on these tasks to a cohort of healthy controls, we may create an individualized profile that identifies where a specific patient falls along an axis of adaptive versus maladaptive functioning within each domain. That multimodal assessment includes diagnostic interviews, questionnaires, behavioral data, and functional neuroimaging. The process begins with a clinical diagnostic interview (e.g., the Structured Clinical Interview for DSM), which is still necessary to validate our emerging results against established clinical practice. The results of that interview and a battery of self-report questionnaires then guide our analysis of imaging and psychophysical data. Figure 2 presents transdiagnostic assessment results for two patients who completed our protocol, compared against a normative database of seventeen healthy controls who completed the same fMRI protocol and who had no psychiatric or medical disorders by self-report. (See SI Methods for more detail on fMRI acquisition and analysis.) Both patients had primary diagnoses of bipolar disorder and were in a major depressive episode. Patient 1 had an additional diagnosis of GAD, and Patient 2 had an additional diagnosis of PTSD. Both showed deviations from normal functioning on measures of emotion regulation and cognitive flexibility. Patient 2, however, had higher negative affect scores, whereas Patient 1 was within population norms. The reverse was true for measures of positive affect and reward motivation. These differences highlight the heterogeneity and functional overlap within a single syndrome such as clinical depression.

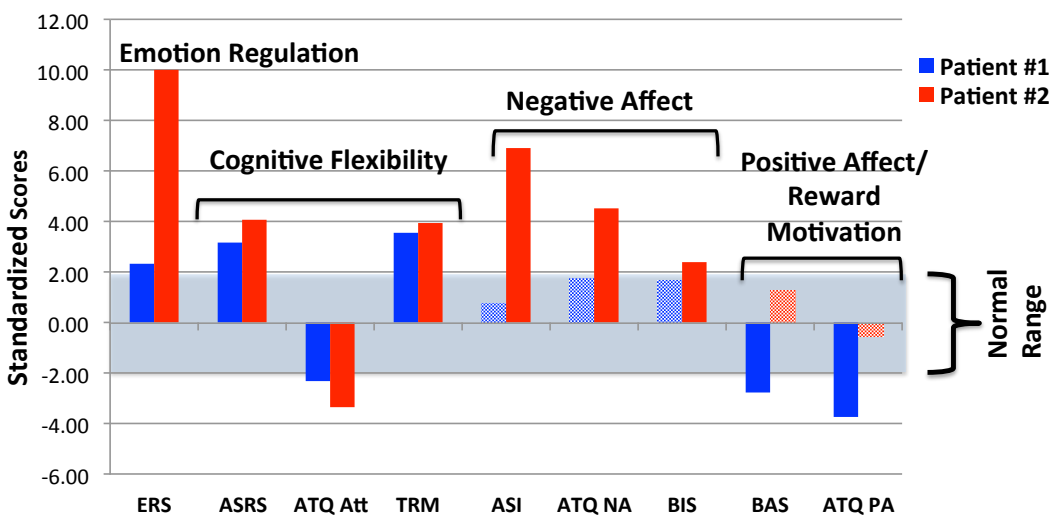

Figure 2: Transdiagnostic psychological assessment for two patients diagnosed with bipolar depression. Shaded bar represents normal range, within 2 standard deviations from standardized means of healthy control cohort. Abbreviations as per Table 1.

\section{Phenotyping Psychiatric Patients with Transdiagnostic Tasks and Imaging}

Behavioral phenotypes may help classify patients, but do not show where or how to intervene. Intervention requires identification of the brain network(s) that drive dysfunction in each domain, which can then be targeted with brain stimulation. We plan to localize those networks spatially with functional magnetic resonance imaging (fMRI) and temporally with magnetoencephalography/electroencephalography (MEG/EEG). Our example patients both showed difficulty with emotion regulation, as measured by the Emotional Reactivity Scale 
(ERS). A well-defined task for measuring this is the Emotional Conflict Resolution (ECR) task $[48,57,58]$. The ECR was developed as an affective analogue of the Stroop task. Subjects are required to report on the emotion expressed by photographed faces (fearful or happy facial expressions) while ignoring a written word appearing as type across the photograph ("Happy" or "Fear"; Figure 3). The written words either match the expression of the face (congruent, or "C" trials, e.g. a fearful face with the word "Fear" written across it) or do not (incongruent, or "I" trials, e.g. a fearful face with the word "Happy" written across it). Performances on trials in this task are history dependent, such that the neural and behavioral response to either a congruent or incongruent image is affected by the image presented immediately prior. These differences represent the recognition of emotion conflict when a congruent trial is followed by an unexpected incongruent trial, or the regulation of emotion conflict when incongruent images are presented one after another. Different neural circuits are recruited during emotion detection based on the presence or absence of emotional conflict in the prior trial [50]. The task therefore models patients' ability to regulate context-irrelevant affective information in the service of meeting ongoing goal-directed demands.

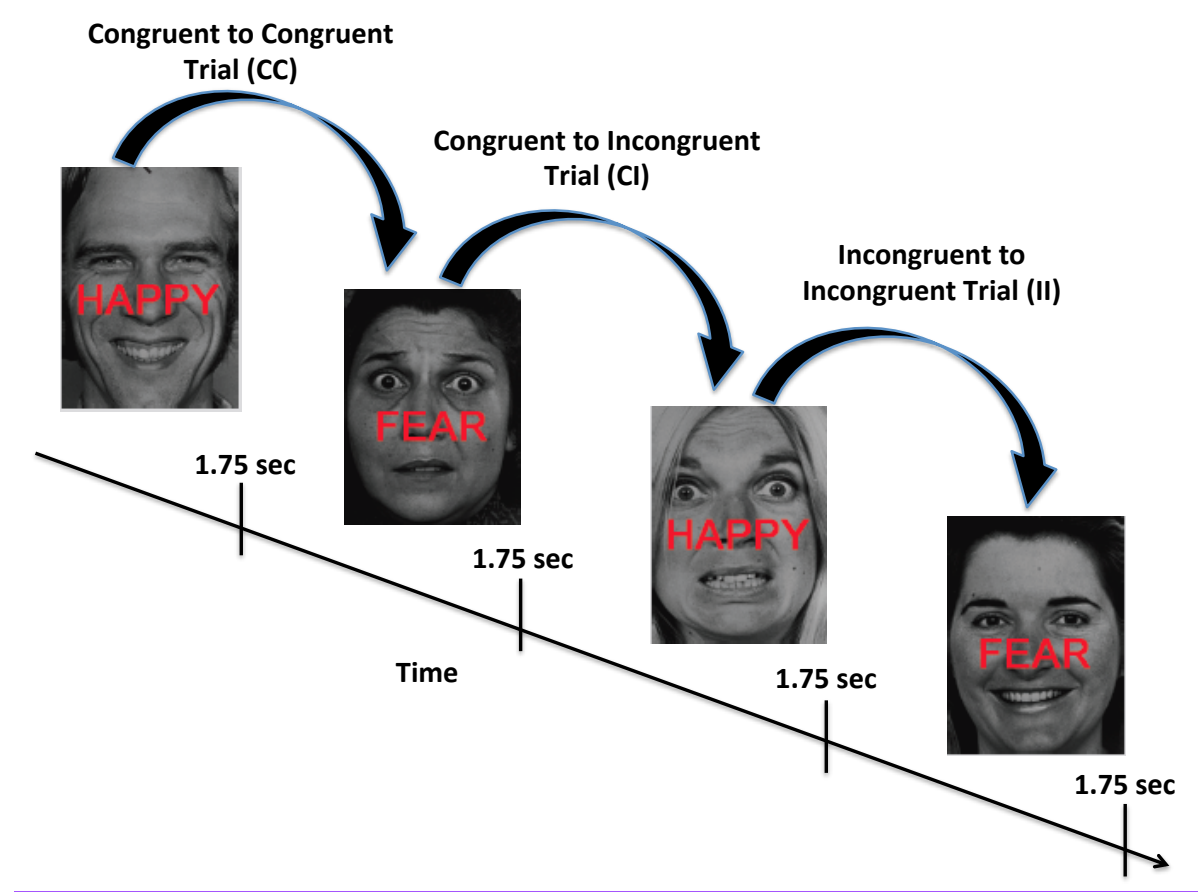

Figure 3: Trial and trial-switch types in the Emotion Conflict Resolution (ECR) task [57]. Subjects judge the facial emotion and attempt to ignore the word during Congruent (C) trials and incongruent (I) trials. Switching from congruent to incongruent (CI) invokes top-down networks for conflict recognition, whereas sequential incongruent trials (II) depend on networks of emotion and conflict regulation.

The ECR task has been validated in multiple fMRI studies. Healthy individuals show greater reaction times in response to incongruent relative to congruent trials, indicating an expected interference effect of emotion conflict. In addition, healthy individuals exhibit slower reaction times on conflict recognition (CI) trials in which they first encounter the "surprise" of incongruence, compared to conflict regulation (II) trials in which this initial response to conflict is overcome in order to continue meeting task demands. Slower reaction times during conflict recognition are associated with additional top-down effort, reflected in greater recruitment of the 
amygdala, dorsomedial prefrontal cortex (dmPFC), and dorsolateral prefrontal cortex (dlPFC) during conflict recognition (CI trials). By contrast, conflict regulation (II) trials are associated with greater regulatory recruitment of the rostral anterior cingulate cortex (rACC) and increased negative coupling between rACC and amygdala, with increases in rACC activity predicting decreases in amygdala activity, indicating successful regulation of emotion conflict.

As an example of a domain-based imaging diagnostic, we used these prior results to develop an ECR-based profiling of emotion regulation in two psychiatric patients. Below, we present a "first-pass" look at single-patient data from these patients as a proof of concept of this approach. Optimizing this for a statistically rigorous comparison of single-patient activations against a cohort of healthy controls is a focus of ongoing work.

Figure 4 shows first-level conflict recognition (CI) > conflict regulation (II) contrasts, computed from a general linear model (GLM; see SI methods). In this preliminary inspection, both patients deviated from activation patterns found in healthy controls, but did so in unique ways (Figure 4a). For example, during conflict recognition trials as compared to conflict regulation, healthy controls show robust bilateral recruitment of dmPFC, dlPFC, vlPFC and anterior insula. The majority of healthy controls to date $(>60 \%)$ have also shown increased amygdala activation in the CI > II contrast, suggesting more effective down-regulation of the amygdala during conflict regulation trials relative to conflict recognition (Figure 4b). Neither Patient 1 nor Patient 2 show this differential $\mathrm{CI}>\mathrm{II}$ amygdala activation, suggesting a failure to adaptively regulate amygdala response during repeated incongruent trials. Patient 1 shows less pronounced vlPFC, dmPFC, and $\mathrm{dACC}$ recruitment, and more pronounced dlPFC recruitment during conflict recognition trials relative to conflict regulation trials. Patient 2 shows increased dmPFC and insula recruitment during conflict recognition trials relative to conflict regulation trials, similar to healthy controls. That patient also had weaker dIPFC and increased rACC recruitment during conflict regulation relative to conflict recognition, opposite to patterns seen both in our cohort of healthy controls and in the literature.

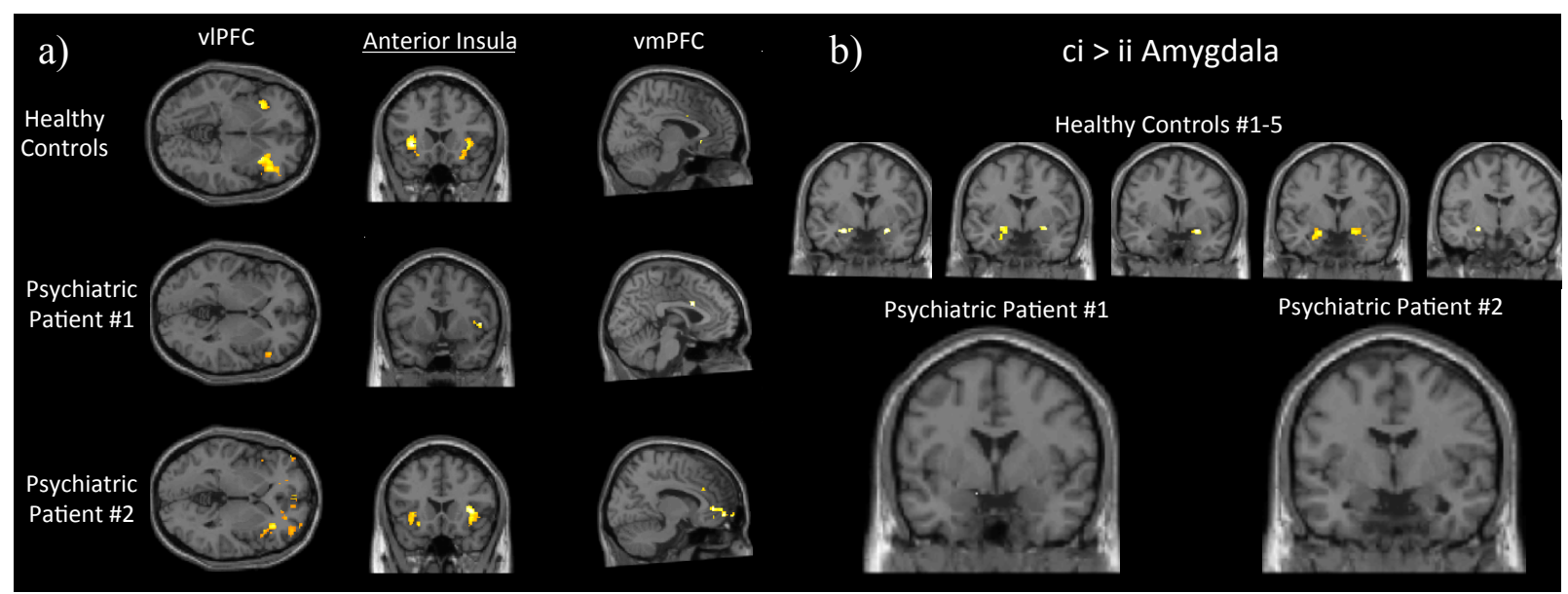

Figure 4 :CI>II contrast results. (A) generalized linear model (GLM) results for healthy controls and individual GLM fixed effects for two psychiatric patients. (B) individual GLM fixed effects in amygdala activation for five representative healthy controls and two psychiatric patients. Highlighted voxels indicate $\mathrm{p}<0.05$ uncorrected. 
We next used psychophysiological interaction (PPI) analyses to identify network changes that may drive patients' behavior. In the recognition vs. regulation (CI $>$ II) contrast, we found a broad pattern of weaker connections among rACC, vmPFC, dlPFC, vlPFC, and dmPFC in both psychiatric patients relative to those found in healthy controls. Functional connectivity patterns also varied between the two patients. For example, both patients show weaker vmPFC-amygdala connectivity than connectivity in healthy controls, but Patient 2 shows substantially weaker functional connectivity than Patient 1 (Figure 5A). Patient 2, by contrast, shows stronger dlPFCamygdala functional connectivity than that seen in healthy controls, while Patient 1 has an opposite pattern (Figure 5B). This illustrates our point that patients with the same categorical diagnosis may have diametrically opposite brain impairments, necessitating a new approach to classifying their specific circuit deficits.

These results suggest the feasibility of transdiagnostic patient classification, but are not sufficient to create a closed-loop system. The high spatial resolution of MRI shows where in the brain a behaviorally-relevant neural dysfunction may reside, but the relatively poor temporal resolution does not reveal the what or when of this dysfunction. That requires a deeper understanding of the temporal dynamics within and between distributed brain regions. Under TRANSFORM, we are collecting that information through invasive human recordings and MEG/EEG in non-surgical patients. The following section will illustrate how those time-resolved techniques might link with fMRI to yield a deeper picture of emotion regulation.
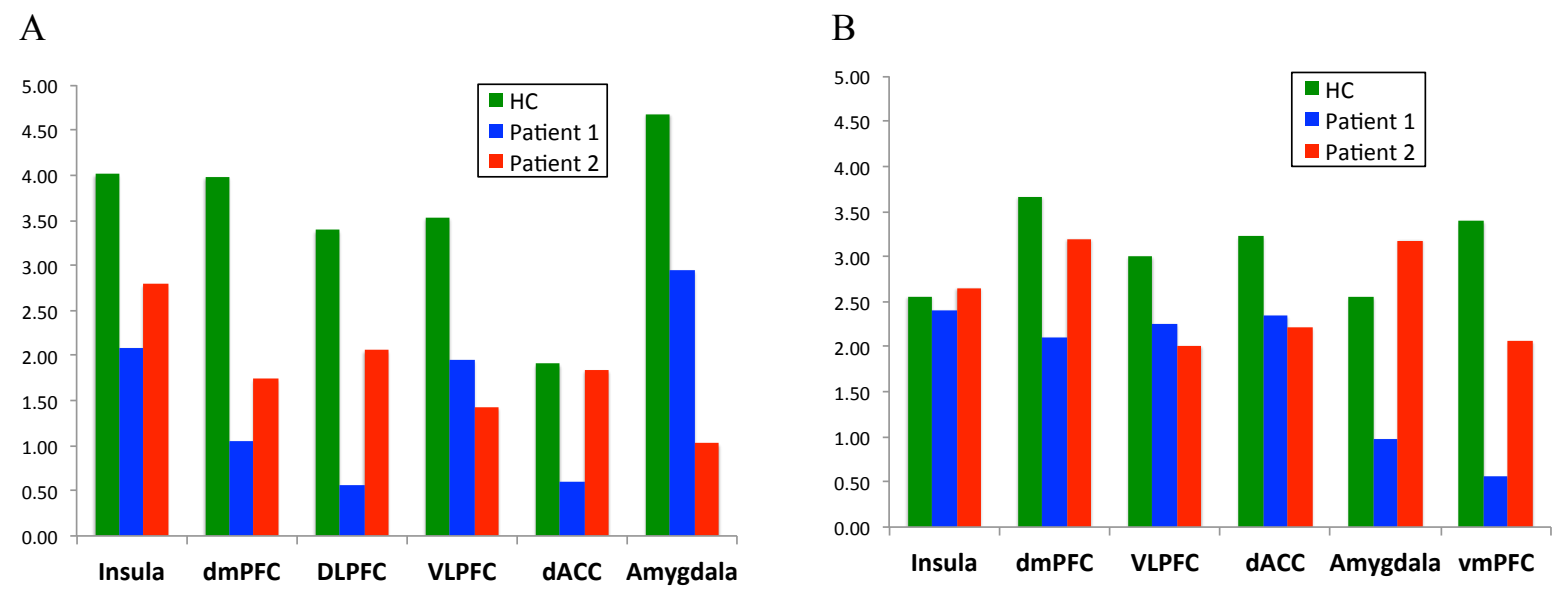

Figure 5: Functional connectivity from fMRI imaging. Results show psychophysiological interaction z-scores for the CI>II contrast in brain regions of interest. (A) vmPFC seed region and (B) dlPFC seed region.

\section{Linking "Where" to "What" Via Human Invasive Electrophysiology}

Closed-loop brain stimulation requires an understanding of how behavior and/or psychological states are encoded moment-to-moment in brain activity, which cannot be captured in fMRI's single snapshots. Electrophysiology can show us "what" neural ensembles are doing at the locations "where" the fMRI results have detect emotional conflict processing. These physiological dynamics need to be understood to modulate the network and identify therapeutically relevant states. Here, we illustrate those dynamics using human LFPs recorded with sub-acutely implanted electrodes during clinical evaluation of patients with intractable epilepsy [59]. Such data have been used to study the mechanisms of a wide variety of cognitive 
capabilities [59-61]. TRANSFORM uses those recordings and our transdiagnostic task battery to link imaging to physiology.

Electrode placement, although driven purely by clinical criteria, often targets the same areas implicated by our trans-diagnostic battery. This allows us to understand LFP dynamics in regions indicated by fMRI. In one prototypical example, a 55 year old woman whose epilepsy was thought to be related to a left insular dysplasia performed the ECR task while awaiting seizure localization (Figure 6). Electrode locations were reconstructed based on post-operative CT scans co-registered with preoperative MRI [62], verifying that we had accessed areas showing ECRrelated fMRI activation in our healthy control cohort (Figure 6A). To examine event-related potentials (ERPs), intracranial LFPs were downsampled from $30 \mathrm{kHz}$ to $1 \mathrm{kHz}$. Line noise (60, 120 , and $180 \mathrm{~Hz}$ ) was removed by channel-wise band-pass filtering and subtraction. Trials with excessive artifact were rejected by visual inspection. The average ERP across trials and its standard error were then computed channel-wise.

Regions implicated by fMRI also showed significant ERP modulation in response to the ECR stimuli. For example, in dmPFC, a window of approximately 1000 to $500 \mathrm{~ms}$ before the response had significant conflict-regulation (II $>\mathrm{CI}$ ) differences (Figure 6B; one-way ANOVA; $\mathrm{n}=7$ II trials; $n=20 \mathrm{CI}$ trials, false discovery rate corrected for multiple timepoints). This is opposite in sign to the fMRI finding, suggesting that the BOLD signal may represent metabolic activity from inhibitory processes during conflict recognition (CI sequences). We did not observe similar large windows of ERP difference in a stimulus-locked ERP analysis (Figure 6B). To examine this effect across regions, we computed the (CI - II) difference for ERPs at multiple electrodes in fMRI-identified regions (Figure 6C). To correct for multiple comparisons across time and space, we computed the null distribution of this multichannel difference by randomly permuting the trial labels 1000 times and converting the differences into a Z-score (Figure 6C). We found strong responses $(Z>1.96, p<0.05)$ in the insula, amygdala, cingulate, vlPFC, vmPFC, dlPFC, and dmPFC. We therefore found a correspondence between neurophysiological activity, in the form of ERPs, and our fMRI findings (Figure 4). This is somewhat surprising, as ERP and fMRI activations do not necessarily arise from the same mechanism, although they may have some common generators [63-65]. These results nevertheless suggest that "where" the neural activity occurs can be mapped with the fMRI and then resolved at the neural population level with LFP.

Changes in LFP network dynamics during ECR (Figure 7) also localize to the same structures implicated by PPI analyses (Figure 5). We examined LFP network connectivity by measuring the trial-averaged coherence within frequency bands and between all pairs of channels [66].

Focusing on the identified significant window of 1000 to $500 \mathrm{~ms}$ before the reaction time, we computed the (CI - II) difference, estimated a null distribution of that difference by 1000 random permutations of the trial labels, and converted the observed coherence to a Z-score against that null distribution. In this patient, we observed band-specific, trial-driven differences in connectivity (Figure 7). II trials had greater coherence at 8-15 Hz (alpha band) between cingulate, amygdala, insula and dIPFC (Figure 7B). CI trials also had increased coherence between frontal structures and insula, but in the high-gamma $65-100 \mathrm{~Hz}$ band (Figure 7E). We thus identify the same structures called out in preliminary fMRI analyses, but with an interesting additional possibility: different frequency bands may serve top-down vs. bottom-up communication, or may reflect rule performance (II) as opposed to rule switching (CI). Other 
investigators have found rule-switching effects in PFC in the alpha-band, although linked to local oscillatory power, not coherence [54].

Overall, these findings suggest that network impairments detected with non-invasive assessment correlate to areas with significant electrophysiologic modulation in response to the same task. It should therefore be possible to use the behavioral and imaging pipelines to design a patientspecific set of implant targets to maximize signal detection for closed-loop applications.

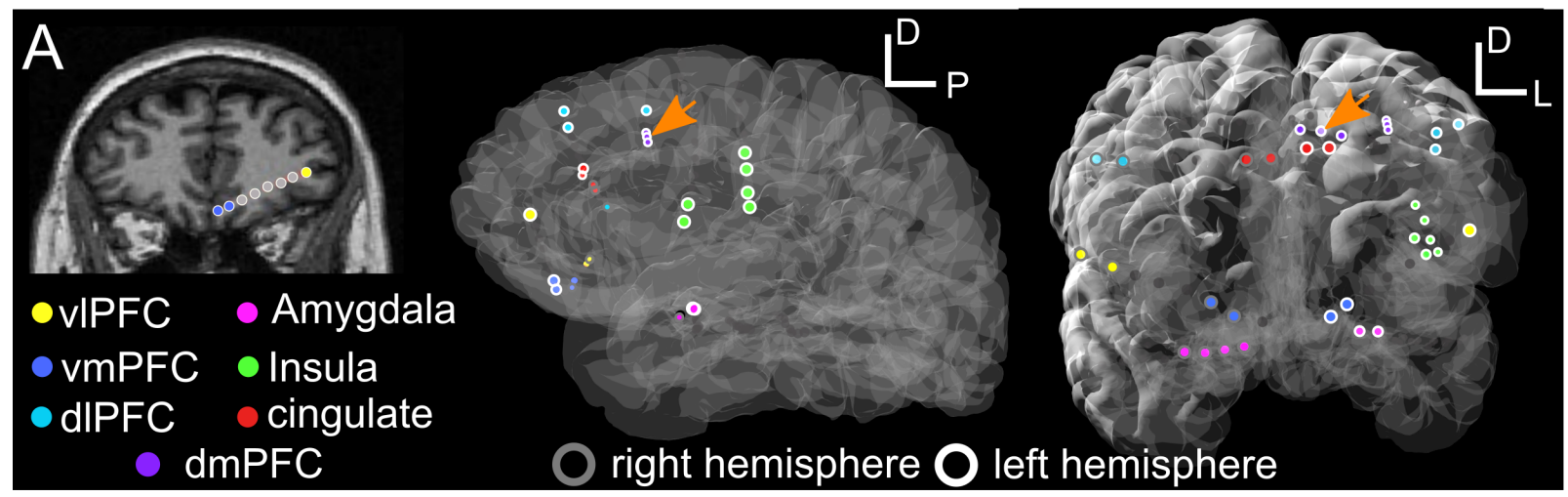

\section{B dmPFC}

Aligned to image onset

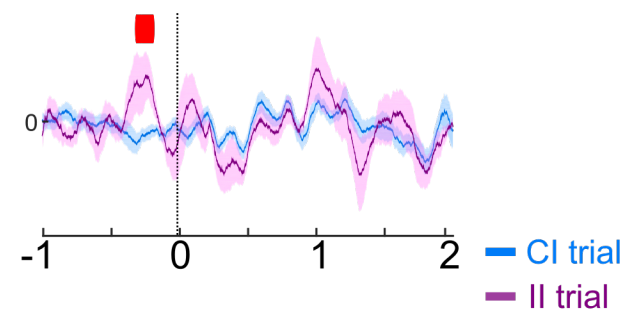

Aligned to reaction time $p$-value $<0.05$

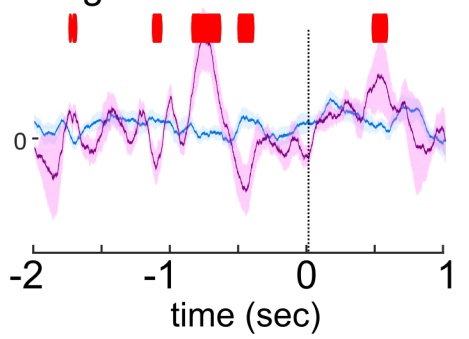

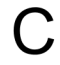

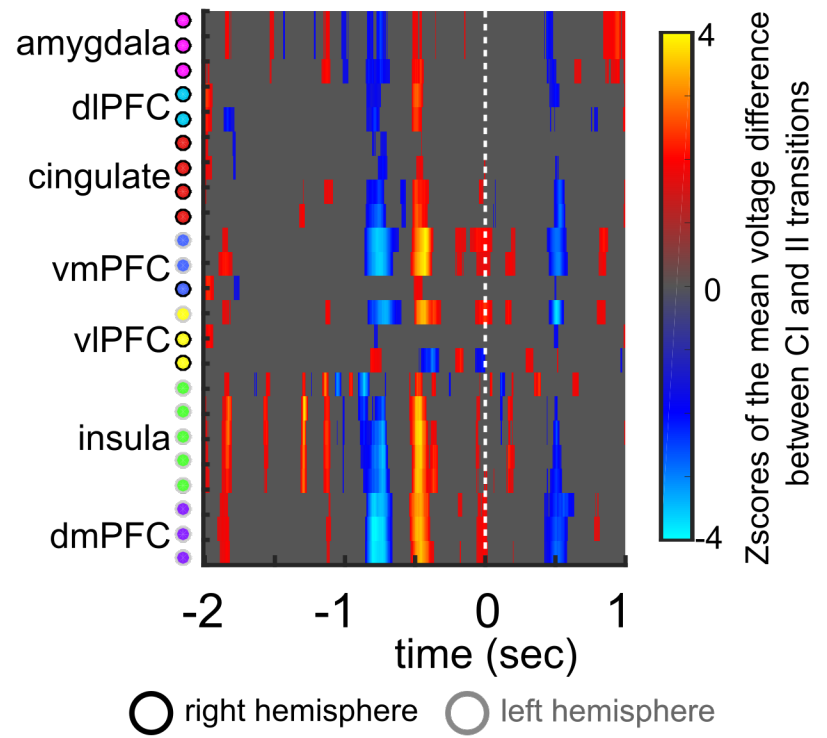

Figure 6: Invasive human electrophysiology during the ECR task. (A), 3-dimensional reconstructions of clinical depth electrodes from fusion of preoperative MRI and postoperative CT. For clarity, the sagittal and coronal views show only those electrodes that correspond to regions identified in fMRI analyses. Colors indicate different brain regions as per the legend. D-dorsal, L-lateral, P-posterior. (B), event-related potentials (ERPs) in the dmPFC, timelocked to stimulus onset (upper) or response (lower). Electrode is indicated by an arrowhead in (A). Red dots indicate significant differences between II and CI trials, false discovery rate controlled per channel. (C), Z-score of (CI - II) ERP difference across brain regions, time-locked to response (white dashed line), masked to show only $|\mathrm{Z}|$ $>1.5$. The period between 1000 and $500 \mathrm{~ms}$ before the response shows strong ERP differences across channels, particularly in insula and medial PFC bilaterally. 

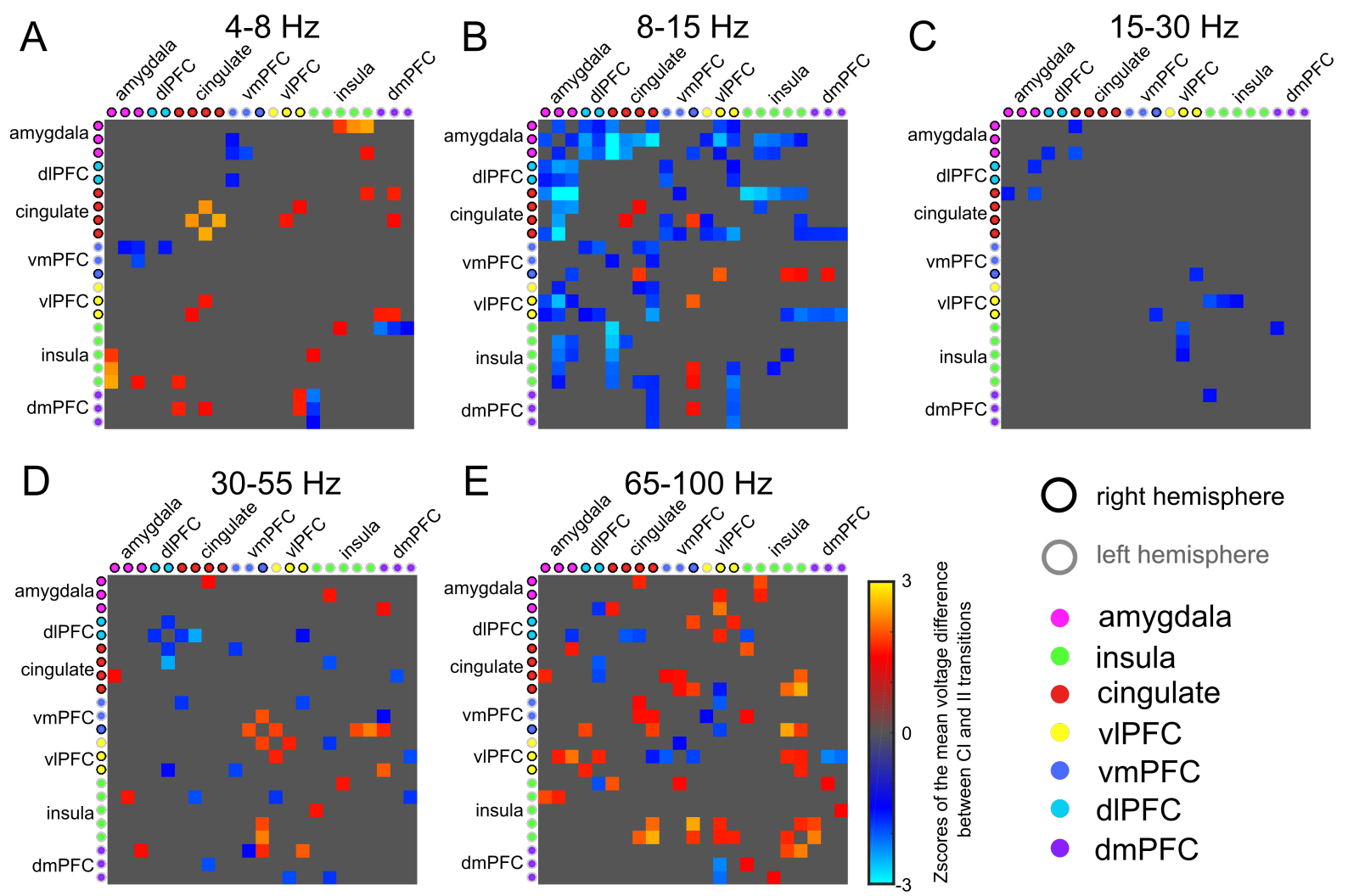

right hemisphere
left hemisphere
amygdala
insula
cingulate
vIPFC
vmPFC
dIPFC
dmPFC

Figure 7. Coherence between channel pairs is frequency-specific during the ECR task. Coherence for each pair is represented as a permutation-derived Z-score of the mean coherence difference between CI and II trials from 1000 to $500 \mathrm{~ms}$ before the button-press response. All values are masked at $|\mathrm{Z}|>1.5$ to emphasize differences. (A) 4-8 Hz, (B) 8-15 Hz, (C) $15-30 \mathrm{~Hz}$, (D) $30-55 \mathrm{~Hz}$, (E) 65-100 Hz. II trials showed higher fronto-insular coherence in the alpha frequency band (B). In contrast, CI trials had higher coherence values in the same regions but in the high gamma band. The dots along the $\mathrm{x}$ and $\mathrm{y}$ axes indicate brain regions based on color and whether the channels were un the left or right hemisphere (see legend).

\section{Modeling the Effects of Brain Stimulation Interventions}

The MRI and LFP data indicate that a single task can engage multiple brain regions in complex ways and may evoke behavior that depends on past trials. Closed-loop control requires lowerdimensional mathematical models of how brain and behavior respond to external drivers such as brain stimulation. In this section, we develop a model to predict the network effects of DBS-like stimulation. We will then link that to behavioral change in the next section.

Stimulation effects can be modeled in causal dynamical systems frameworks [67-69], from linear multivariate autoregressive (MVAR) models to non-linear dynamic causal models [70,71]. Such models may capture differences in network "wiring" under different trial conditions, including the presence/absence of brain stimulation. Here, we present an example using a general linear multivariate (gMVR) network model. A gMVR is of the form:

$$
Y=X \beta+E
$$

Where, $Y$ is the series of multivariate measurements, $X$ is the matrix of predictor variables, $\beta$ is the matrix of parameters to be estimated, and $E$ is multivariate white noise. Similar models form the basis of Granger causality analysis [72-74]. A standard MVAR model, however, uses a fixed and low-order structure (generally 5-10 lags) for both the local activity within a brain structure (self history) and the propagation of activity between structures (cross history). This is not 
biophysically correct, as both axonal conduction and synaptic transmission can introduce tens of milliseconds of delay between areas. Hence, we adapted the framework to have a short-term self history and longer, independently chosen time lags for cross history.

We fit this gMVR model using LFP data from the right vmPFC, dACC, and amygdala in the same patient as Figure 6, incorporating a simple forcing function representing stimulation at each node (SI Methods). The model predicts different effects depending on both the stimulated node and the trial type (Figure 8A). Perturbing vmPFC or dACC during CI trials is overall less effective than stimulating during II trials. dACC stimulation (increase in gamma power) tends to inhibit vmPFC regardless of the trial type, whereas the effects of amygdala or VmPFC stimulation can be positive, negative, or neutral depending on recent trial history.

This basic model shows evidence of being able to predict stimulation outcomes. It suggests that the strongest effect would be found from dACC stimulation, which should inhibit both vmPFC and amygdala, leaving the patient unable to effectively process stimuli or regulate emotion. On a second experimental day with the same patient, we delivered high-frequency stimulation to dACC or amygdala on 50\% of ECR trials just after the stimulus appeared (SI Methods). dACC stimulation produced a reaction time increase, consistent with the network disruption predicted by the gMVR model (Figure 8B).
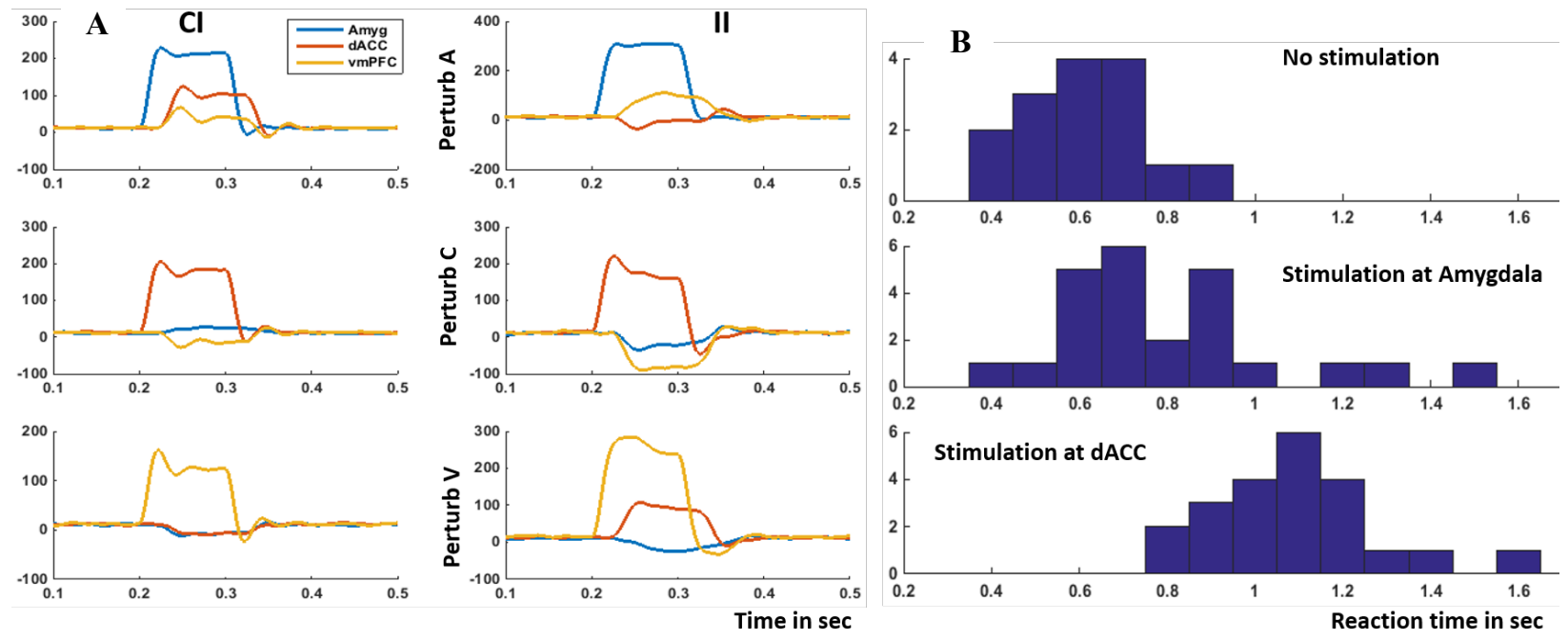

Figure 8: General multivariate regression models for simulating band limited LFP power. (A), Simulation of the effects of perturbation (in the form of an additive term to one of the nodes) at amygdala (blue, A), dACC (red, C) and vmPFC (yellow, V) for CI and II trials. (B), reaction times of CI trials with no stimulation and with $160 \mathrm{~Hz} / 6$ $\mathrm{mA}$ stimulation at $\mathrm{A}$ and at $\mathrm{C}$.

Based on these encouraging results with a relatively simple model, we hope to achieve more robust and wide-ranging predictions by expanding it to more detailed biophysics. Another future direction will extend the behavioral result, seeking a relationship between specific neural changes (spectral/time domain) and behavior. By reversing that relationship, we might predict what needs to be changed in the neural domain (via stimulation) to steer the network's behavioral output towards a therapeutic target. 


\section{State-Space Behavior Modeling to Capture Effects of Brain Stimulation}

Brain stimulation in this emotion-regulation network does more than simply increase reaction time (RT). Figure 9 shows the reaction time effect of Figure 8B at the single trial level. Both RT and fraction of trials correct change in response to both stimulation and the task factors.

Stimulation does not differentially affect CI and II trials (Figures 9B, 9C), but does cause trials with a Fear $(\mathrm{F})$ valence to have lower performance and higher reaction times under stimulation. This effect may be more pronounced for dACC stimulation. There also appears to be a general trend superimposed on each block's effect. For instance, RT in the fourth block (second amygdala stimulation experiment) rises sharply in the beginning of the block, then drops.

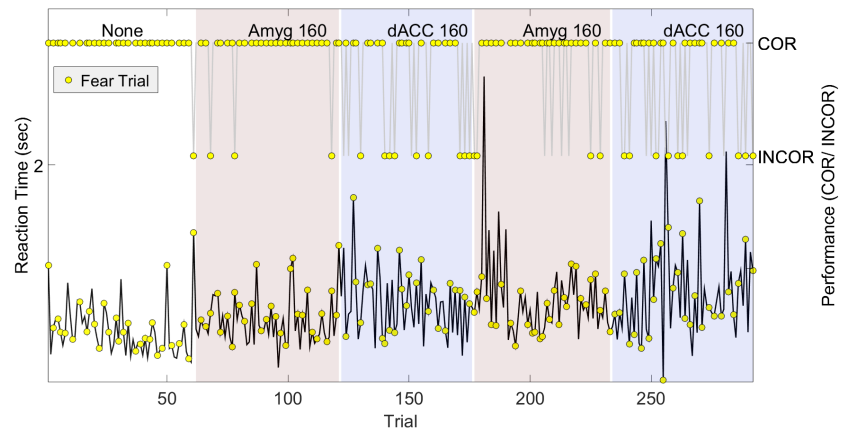

A

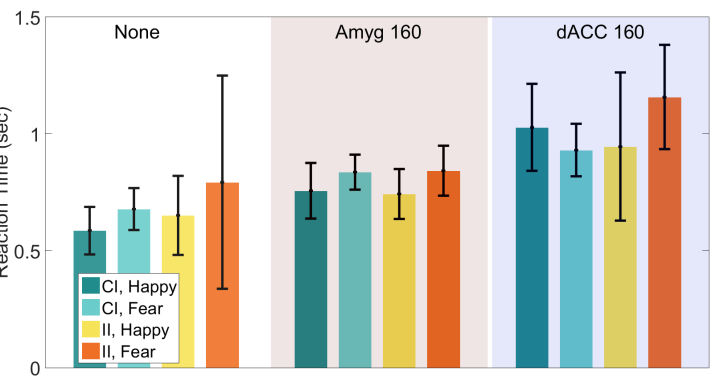

$$
\begin{array}{ll}
12 \\
C^{2}
\end{array}
$$

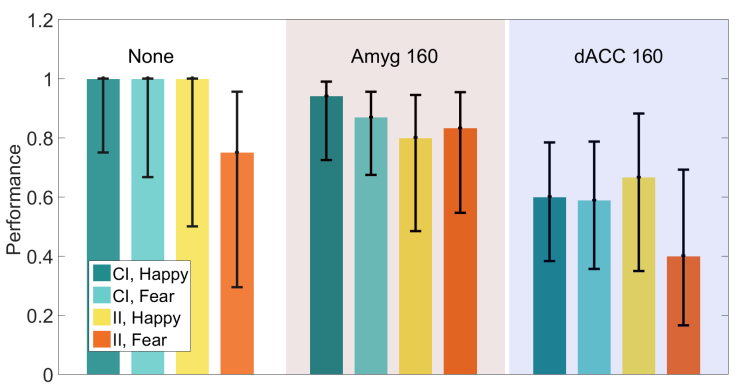

Figure 9: Behavioral summary of ECR under brain stimulation. (A), reaction time and performance (fraction correct) at the single-trial level with multiple sites of stimulation. (B), reaction time per stimulation block. (C), performance per stimulation block. Broadly, stimulation appears to affect trials with a fearful face more than trials with a happy face, regardless of site. CI trials do not appear to differ from II trials in reaction time or performance unless a fearful face is present.

These time-varying behavioral effects and interactions can be summarized in a low-dimensional representation. State-space models consider multiple outputs (e.g., RT and performance) to be common reflections of an underlying hidden state. The state-space approach has successfully modeled low-dimensional dynamic features of both neural and behavioral signals for a variety of cognitive tasks, including learning and Stroop-like tasks [75-79]. Here, we define the state as the patient's response to F trials (SI Methods). The higher the state, the more the subject is affected and distracted by fear. When we estimate the maximum-likelihood value of the state process through an expectation-maximization (E-M) algorithm as per [78,79], we obtain a single variable summarizing the effects of brain stimulation on decisions, reaction time, and overall fear responsiveness (Figure 10). With stimulation in either dACC or amygdala, the state increases, tracking an increase in RT and reduction in performance. Interestingly, the result is step-like: any brain stimulation moves the system to a new state that persists even on unstimulated trials. The state-space analysis also confirms the gMVR results of the preceding section: the largest effects arise from stimulation in dACC. 


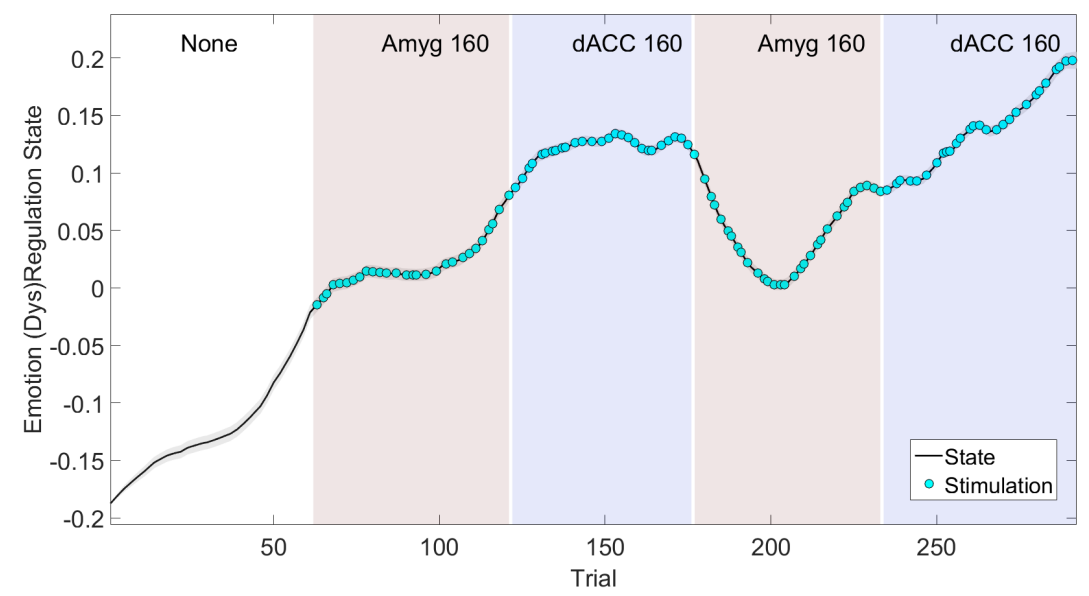

Figure 10: Cognitive state prediction in response to brain stimulation. The state variable, which reflects emotional lability to fear, increases in response to brain stimulation. Amygdala generally causes smaller changes than dACC. Both stimulation types appear to cause a step/plateau response in the state, despite being delivered on only half of the available trials.

More importantly, this approach is not limited to ECR or Gaussian-distributed variables. In [79], we describe an extension to gamma-distributed data. The behavioral output of all six domainlinked tasks can be tracked through this latent-variable approach once a theoretically valid model is formulated. This should make neural decoding for closed-loop control substantially simpler: the state variable substitutes for the velocity/position seen in a traditional brain-computer analysis, with similarly smooth temporal dynamics.

\section{Discussion and Conclusion}

In our vision of next-generation psychiatric DBS, a patient will proceed through a clinical workup based on the transdiagnostic framework (Figure 11), and their brain stimulator will be targeted and programmed based on that framework. We have shown preliminary evidence that this pipeline is possible. This begins with neuro-imaging and behavioral assessment. In our framework, two patients who had the same clinical diagnosis (depressive episode) were more clearly separable in terms of their specific impairments. In a different patient, pathology might not take the form of visibly impaired behavior. Instead, it may appear as "compensatory activation" in electro-magnetic imaging, where patients use a wider set of brain areas (and thus detract from other ongoing processing) to maintain behavioral performance.

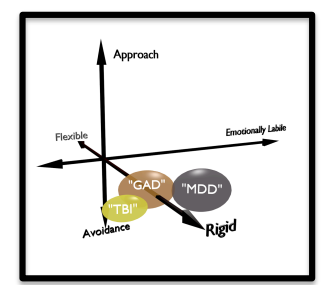

A. Classify

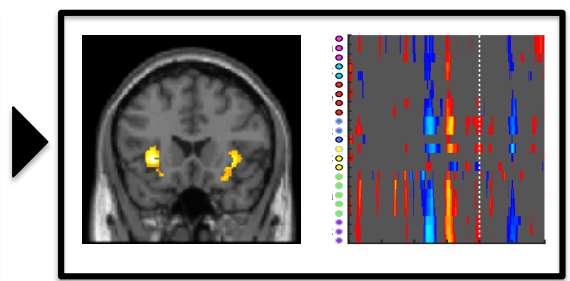

B. Localize in Space \& Time

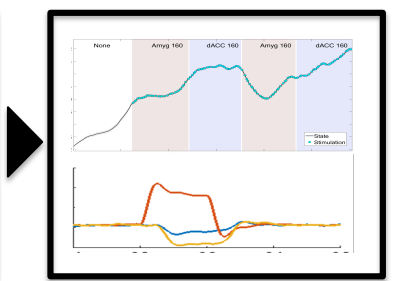

C. Model \& Identify

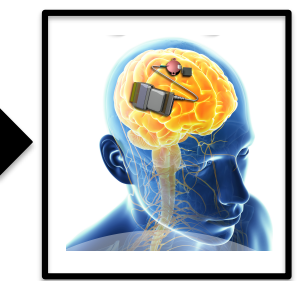

D. Target \& Treat

Figure 11: TRANSFORM DBS pipeline. An individual patient's dysfunction is classified in trans-diagnostic space (A). The associated brain activations are localized (B), and their relation to behavior identified through cognitive state modeling $(\mathbf{C})$. Those models also predict the brain and behavioral response to applied perturbations such as 
electrical stimulation. From this assessment, we hope to design a patient-specific closed-loop therapy to be delivered through an integrated, multi-point closed-loop system (D).

To link those activations to a patient-customized closed-loop controller, we must move from the spatial (anatomic) to the temporal domain. A key aspect of TRANSFORM is behavioral assessment while measuring brain activation with multiple modalities, both invasive and noninvasive. We showed an example from one of our first recordings in the epilepsy monitoring unit, where the same brain areas that showed high BOLD signal were recorded in a human patient with electrodes that had been implanted for seizure location. ERP and coherence analyses found changes in the same areas identified with fMRI, but resolved them to a particular time window relative to the response and to specific frequency bands.

Controlling those electrical and behavioral signatures requires a model of the "transfer function" between the brain's inputs (task cues and stimulation) and its outputs (symptoms and behavior). We presented two complementary routes toward this understanding. First, we showed that through a gMVR method, we may identify differences in brain network structure that correlate to task performance. Second, we presented initial progress toward a low-dimensional state-space approach to modeling the behavioral effects of stimulation. Expressing control in this state-space framework may simplify the process of controller development, since it allows us to simultaneously account for the effect of multiple task variables on multiple behavior outputs. In particular, we showed that amygdala and cingulate stimulation both affect behavior, but in different ways that are captured by the state-space model. While this example showed a behavioral impairment (increased reaction time and decreased number of correct trials), the same framework could capture behavioral improvement caused by stimulation at another site.

The final challenge is bringing these components together into a usable clinical device. That requires not only neural decoding and control algorithms based on these findings, but hardware that can embody those algorithms and pass regulatory approval. Given the effort and expense of qualifying implantable hardware for human use, it would be very desirable if that platform were sufficiently flexible to enable a variety of future studies. Unfortunately, no current clinical DBS system can access more than two sites at once, and stimulation linked to recordings remains an investigational feature $[80,81]$. To bring our work to the clinic, we are collaborating with a multidisciplinary engineering team to design a next-generation DBS system. As described more fully elsewhere [82,83], this platform will be capable of stimulating four different brain sites while recording from all of those plus an optional fifth site. It includes a reconfigurable processing architecture suitable for implementing these algorithms. Power and battery will continue to be driving concerns, but careful choice of neural features should mitigate this.

In summary, although DBS for mental illness has faced hurdles, we believe the future is bright. There are major opportunities to re-consider the design and targeting of those systems now that the open-loop strategy has been proven inadequate. The TRANSFORM framework offers a way forward, based on an emerging understanding that psychiatric impairment often does not respect diagnostic boundaries. Our work in coming years will test the preliminary findings above and refine them for eventual clinical use. 


\section{Acknowledgements}

We thank Mr. David Clifford and Dr. Geoffrey Ling for helpful comments on an earlier draft of the manuscript. ACP, ASW, AY, DDD, ENE, KKE, TD, and UTE are named inventors on patent applications related to transdiagnostic tasks and their use in calibrating brain stimulation. DDD and ENE have served as paid consultants to Medtronic and Cyberonics, which manufacture clinical brain stimulators. This research was sponsored by the U.S. Army Research Office and the Defense Advanced Research Projects Agency and was accomplished under Cooperative Agreement Number W911NF-14-2-0045. The views and conclusions contained in this document are those of the authors and should not be interpreted as representing the official policies, either expressed or implied, of the Army Research Office, DARPA, or the U.S. Government. The U.S. Government is authorized to reproduce and distribute reprints for Government purposes notwithstanding any copyright notation hereon. 


\section{References Cited}

[1] Whiteford HA, Degenhardt L, Rehm J, Baxter AJ, Ferrari AJ, Erskine HE, et al. Global burden of disease attributable to mental and substance use disorders: findings from the Global Burden of Disease Study 2010. The Lancet 2013;382:1575-86. doi:10.1016/S01406736(13)61611-6.

[2] Kessler RC, Heeringa SG, Stein MB, et al. Thirty-day prevalence of DSM-IV mental disorders among nondeployed soldiers in the US Army: Results from the Army Study to Assess Risk and Resilience in Servicemembers (Army STARRS). JAMA Psychiatry 2014;71:504-13. doi:10.1001/jamapsychiatry.2014.28.

[3] Reger MA, Smolenski DJ, Skopp NA, et al. RIsk of suicide among us military service members following operation enduring freedom or operation iraqi freedom deployment and separation from the us military. JAMA Psychiatry 2015;72:561-9. doi:10.1001/jamapsychiatry.2014.3195.

[4] Insel MD Thomas. Assessing the economic costs of serious mental illness. Am J Psychiatry 2008;165:663-5. doi:10.1176/appi.ajp.2008.08030366.

[5] Insel TR. Disruptive insights in psychiatry: transforming a clinical discipline. J Clin Invest 2009;119:700-5. doi:10.1172/JCI38832.

[6] Lieberman JA, Stroup TS, McEvoy JP, Swartz MS, Rosenheck RA, Perkins DO, et al. Effectiveness of antipsychotic drugs in patients with chronic schizophrenia. N Engl J Med 2005;353:1209-23. doi:10.1056/NEJMoa051688.

[7] Warden D, Rush AJ, Trivedi MH, Fava M, Wisniewski SR. The STAR*D project results: A comprehensive review of findings. Curr Psychiatry Rep 2007;9:449-59. doi:10.1007/s11920-007-0061-3.

[8] Gaynes B, Warden D, Trivedi MH, Wisniewski S, Fava M, Rush AJ. What did STAR*D teach us? Results from a large-scale, practical, clinical trial for patients with depression. Psychiatr Serv 2009;60:1439-45. doi:10.1176/appi.ps.60.11.1439.

[9] Lieberman JA, Stroup TS. The NIMH-CATIE schizophrenia study: what did we learn? Am J Psychiatry 2011;168:770-5. doi:10.1176/appi.ajp.2011.11010039.

[10] Thomas JL, Wilk JE, Riviere LA, McGurk D, Castro CA, Hoge CW. Prevalence of mental health problems and functional impairment among active component and National Guard soldiers 3 and 12 months following combat in Iraq. Arch Gen Psychiatry 2010;67:614-23. doi:10.1001/archgenpsychiatry.2010.54.

[11] Hoge CW, Riviere LA, Wilk JE, Herrell RK, Weathers FW. The prevalence of posttraumatic stress disorder (PTSD) in US combat soldiers: a head-to-head comparison of DSM-5 versus DSM-IV-TR symptom criteria with the PTSD checklist. Lancet Psychiatry n.d. doi:10.1016/S2215-0366(14)70235-4.

[12] Jonas D, Cusack K, Forneris C, Wilkins T, Sonis J, Middleton J, et al. Comparative Effectiveness Review: Psychological treatments and pharmacological treatments for adults with post-traumatic stress disorder (PTSD) 2013.

[13] Powers MB, Halpern JM, Ferenschak MP, Gillihan SJ, Foa EB. A meta-analytic review of prolonged exposure for posttraumatic stress disorder. Clin Psychol Rev 2010;30:635-41. doi:10.1016/j.cpr.2010.04.007.

[14] van den Berg DPG, de Bont PAJM, van der Vleugel BM, de Roos C, de Jongh A, Van Minnen A, et al. Prolonged Exposure vs Eye Movement Desensitization and Reprocessing vs Waiting List for Posttraumatic Stress Disorder in Patients With a Psychotic Disorder: A 
Randomized Clinical Trial. JAMA Psychiatry 2015;72:259.

doi:10.1001/jamapsychiatry.2014.2637.

[15] Insel TR. Faulty Circuits. Sci Am 2010;302:44-51. doi:10.1038/scientificamerican0410-44.

[16] Insel TR, Wang PS. Rethinking mental illness. J Am Med Assoc 2010;303:1970-1. doi:10.1001/jama.2010.555.

[17] Kelly D, Richardson A, Mitchell-Heggs N. Stereotactic limbic leucotomy: neurophysiological aspects and operative technique. Br J Psychiatry J Ment Sci 1973;123:133-40.

[18] Bingley T, Leksell L, Meyerson B, Rylander G. Long-term results of stereotactic capsulotomy in chronic obsessive compulsive neurosis. Neurosurg. Treat. Psychiatry Pain Epilepsy, University Park Press; 1977, p. 287-9.

[19] Ballantine HT Jr, Bouckoms AJ, Thomas EK, Giriunas IE. Treatment of psychiatric illness by stereotactic cingulotomy. Biol Psychiatry 1987;22:807-19.

[20] Rauch SL, Dougherty DD, Cosgrove GR, Cassem EH, Alpert NM, Price BH, et al. Cerebral metabolic correlates as potential predictors of response to anterior cingulotomy for obsessive compulsive disorder. Biol Psychiatry 2001;50:659-67.

[21] Dougherty DD, Baer L, Cosgrove GR, Cassem EH, Price BH, Nierenberg AA, et al. Prospective long-term follow-up of 44 patients who received cingulotomy for treatmentrefractory obsessive-compulsive disorder. Am J Psychiatry 2002;159:269-75. doi:10.1176/appi.ajp.159.2.269.

[22] Greenberg BD, Rauch SL, Haber SN. Invasive circuitry-based neurotherapeutics: stereotactic ablation and deep brain stimulation for OCD. Neuropsychopharmacology 2010;35:317-36. doi:10.1038/npp.2009.128.

[23] Yang JC, Ginat DT, Dougherty DD, Makris N, Eskandar EN. Lesion analysis for cingulotomy and limbic leucotomy: comparison and correlation with clinical outcomes. J Neurosurg 2013. doi:10.3171/2013.9.JNS13839.

[24] George MS, Rush AJ, Marangell LB, Sackeim HA, Brannan SK, Davis SM, et al. A OneYear Comparison of Vagus Nerve Stimulation with Treatment as Usual for TreatmentResistant Depression. Biol Psychiatry 2005;58:364-73. doi:10.1016/j.biopsych.2005.07.028.

[25] Aaronson ST, Carpenter LL, Conway CR, Reimherr FW, Lisanby SH, Schwartz TL, et al. Vagus nerve stimulation therapy randomized to different amounts of electrical charge for treatment-resistant depression: acute and chronic effects. Brain Stimulat 2013;6:631-40. doi:10.1016/j.brs.2012.09.013.

[26] George MS, Lisanby SH, Avery D, McDonald WM, Durkalski V, Pavlicova M, et al. Daily left prefrontal Transcranial Magnetic Stimulation therapy for Major Depressive Disorder: A sham-controlled randomized trial. Arch Gen Psychiatry 2010;67:507-16. doi:10.1001/archgenpsychiatry.2010.46.

[27] Johnson KA, Baig M, Ramsey D, Lisanby SH, Avery D, McDonald WM, et al. Prefrontal rTMS for treating depression: Location and intensity results from the OPT-TMS multi-site clinical trial. Brain Stimulat 2013;6:108-17. doi:10.1016/j.brs.2012.02.003.

[28] Mayberg HS. Targeted electrode-based modulation of neural circuits for depression. J Clin Invest 2009;119:717-25. doi:10.1172/JCI38454.

[29] Malone DA, Dougherty DD, Rezai AR, Carpenter LL, Friehs GM, Eskandar EN, et al. Deep brain stimulation of the ventral capsule/ventral striatum for treatment-resistant depression. Biol Psychiatry 2009;65:267-75. doi:10.1016/j.biopsych.2008.08.029. 
[30] Greenberg B, Gabriels L, Malone D, Rezai A, Friehs G, Okun M, et al. Deep brain stimulation of the ventral internal capsule/ventral striatum for obsessive-compulsive disorder: worldwide experience. Mol Psychiatry 2010;15:64-79. doi:10.1038/mp.2008.55.

[31] Morishita T, Fayad SM, Higuchi M, Nestor KA, Foote KD. Deep brain stimulation for treatment-resistant depression: systematic review of clinical outcomes. Neurotherapeutics 2014;11:475-84. doi:10.1007/s13311-014-0282-1.

[32] Dougherty DD, Rezai AR, Carpenter LL, Howland RH, Bhati MT, O'Reardon JP, et al. A randomized sham-controlled trial of deep brain stimulation of the ventral capsule/ventral striatum for chronic treatment-resistant depression. Biol Psychiatry 2015;78:240-8. doi:10.1016/j.biopsych.2014.11.023.

[33] Garnaat SL, Greenberg BD, Sibrava NJ, Goodman WK, Mancebo MC, Eisen JL, et al. Who qualifies for deep brain stimulation for OCD? Data from a naturalistic clinical sample. J Neuropsychiatry Clin Neurosci 2014;26:81-6. doi:10.1176/appi.neuropsych.12090226.

[34] Ward MP, Irazoqui PP. Evolving refractory major depressive disorder diagnostic and treatment paradigms: toward closed-loop therapeutics. Front Neuroengineering 2010;3:7. doi:10.3389/fneng.2010.00007.

[35] Widge AS, Dougherty DD, Moritz CT. Affective brain-computer interfaces as enabling technology for responsive psychiatric stimulation. Brain-Comput Interfaces 2014;1:126-36. doi:10.1080/2326263X.2014.912885.

[36] Morrell MJ. Responsive cortical stimulation for the treatment of medically intractable partial epilepsy. Neurology 2011;77:1295-304. doi:10.1212/WNL.0b013e3182302056.

[37] Rosin B, Slovik M, Mitelman R, Rivlin-Etzion M, Haber SN, Israel Z, et al. Closed-loop deep brain stimulation is superior in ameliorating Parkinsonism. Neuron 2011;72:370-84. doi:10.1016/j.neuron.2011.08.023.

[38] Little S, Pogosyan A, Neal S, Zavala B, Zrinzo L, Hariz M, et al. Adaptive deep brain stimulation in advanced Parkinson disease. Ann Neurol 2013;74:449-57. doi:10.1002/ana.23951.

[39] Whelan R, Garavan H. When optimism hurts: inflated predictions in psychiatric neuroimaging. Biol Psychiatry 2013. doi:10.1016/j.biopsych.2013.05.014.

[40] Widge AS, Avery DH, Zarkowski P. Baseline and treatment-emergent EEG biomarkers of antidepressant medication response do not predict response to repetitive transcranial magnetic stimulation. Brain Stimulat 2013;6:929-31. doi:10.1016/j.brs.2013.05.001.

[41] McLoughlin G, Makeig S, Tsuang M. In search of biomarkers in psychiatry: EEG-based measures of brain function. Am J Med Genet B Neuropsychiatr Genet 2014;165:111-21. doi:10.1002/ajmg.b.32208.

[42] Cuthbert BN, Insel TR. Toward the future of psychiatric diagnosis: the seven pillars of RDoC. BMC Med 2013;11:126. doi:10.1186/1741-7015-11-126.

[43] Widge AS, Deckersbach T, Eskandar EN, Dougherty DD. Deep brain stimulation for treatment-resistant psychiatric illnesses: what has gone wrong and what should we do next? Biol Psychiatry 2015. doi:10.1016/j.biopsych.2015.06.005.

[44] Regier DA, Narrow WE, Clarke DE, Kraemer HC, Kuramoto SJ, Kuhl EA, et al. DSM-5 field trials in the United States and Canada, part II: test-retest reliability of selected categorical diagnoses. Am J Psychiatry 2013;170:59-70. doi:10.1176/appi.ajp.2012.12070999.

[45] American Psychiatric Association. Diagnostic and statistical manual of mental disorders (DSM). 5th ed. Arlington, VA: American Psychiatric Publishing; 2013. 
[46] Milad MR, Pitman RK, Ellis CB, Gold AL, Shin LM, Lasko NB, et al. Neurobiological basis of failure to recall extinction memory in posttraumatic stress disorder. Biol Psychiatry 2009;66:1075-82. doi:10.1016/j.biopsych.2009.06.026.

[47] Rougemont-Bücking A, Linnman C, Zeffiro TA, Zeidan MA, Lebron-Milad K, RodriguezRomaguera J, et al. Altered processing of contextual information during fear extinction in PTSD: an fMRI study. CNS Neurosci Ther 2011;17:227-36. doi:10.1111/j.17555949.2010.00152.x.

[48] Etkin A, Egner T, Kalisch R. Emotional processing in anterior cingulate and medial prefrontal cortex. Trends Cogn Sci 2011;15:85-93. doi:10.1016/j.tics.2010.11.004.

[49] Rive MM, van Rooijen G, Veltman DJ, .Phillips ML, Schene AH, Ruhé HG. Neural correlates of dysfunctional emotion regulation in major depressive disorder. A systematic review of neuroimaging studies. Neurosci Biobehav Rev 2013;37:2529-53. doi:10.1016/j.neubiorev.2013.07.018.

[50] Heller AS, Johnstone ,T, Peterson ,MJ, Kolden, GG, Kalin, NH, Davidson, RJ. Increased prefrontal cortex activity during negative emotion regulation as a predictor of depression symptom severity trajectory over 6 months. JAMA Psychiatry 2013;70:1181-9. doi:10.1001/jamapsychiatry.2013.2430.

[51] Bleich A, Koslowsky M, Dolev A, Lerer B. Post-traumatic stress disorder and depression. An analysis of comorbidity. Br J Psychiatry 1997;170:479-82.

[52] Campbell DG, Felker BL, Liu C-F, Yano EM, Kirchner JE, Chan D, et al. Prevalence of Depression-PTSD Comorbidity: Implications for Clinical Practice Guidelines and Primary Care-based Interventions. J Gen Intern Med 2007;22:711-8. doi:10.1007/s11606-006-01014.

[53] Miller EK, Cohen JD. An integrative theory of prefrontal cortex function. Annu Rev Neurosci 2001;24:167-202.

[54] Buschman TJ, Denovellis EL, Diogo C, Bullock D, Miller EK. Synchronous oscillatory neural ensembles for rules in the prefrontal cortex. Neuron 2012;76:838-46. doi:10.1016/j.neuron.2012.09.029.

[55] Siegel M, Buschman TJ, Miller EK. Cortical information flow during flexible sensorimotor decisions. Science 2015;348:1352-5. doi:10.1126/science.aab0551.

[56] Kehagia AA, Murray GK, Robbins TW. Learning and cognitive flexibility: frontostriatal function and monoaminergic modulation. Curr Opin Neurobiol 2010;20:199-204. doi:10.1016/j.conb.2010.01.007.

[57] Etkin A, Egner T, Peraza DM, Kandel ER, Hirsch J. Resolving emotional conflict: a role for the rostral anterior cingulate cortex in modulating activity in the amygdala. Neuron 2006;51:871-82. doi:10.1016/j.neuron.2006.07.029.

[58] Etkin A, Prater KE, Hoeft F, Menon V, Schatzberg AF. Failure of anterior cingulate activation and connectivity with the amygdala during implicit regulation of emotional processing in generalized anxiety disorder. Am J Psychiatry 2010;167:545-54.

[59] Lachaux J, Rodriguez E, Martinerie J, Adam C, Hasboun D, Varela F. A quantitative study of gamma-band activity in human intracranial recordings triggered by visual stimuli. Eur $\mathbf{J}$ Neurosci 2000;12:2608-22. doi:10.1046/j.1460-9568.2000.00163.x.

[60] Dykstra AR, Halgren E, Thesen T, Carlson C, Doyle E, Madsen JR, et al. Widespread Brain Areas Engaged during a Classical Auditory Streaming Task Revealed by Intracranial EEG. Front Hum Neurosci 2011;5:1-14. doi:10.3389/fnhum.2011.00074. 
[61] Chan AM, Dykstra AR, Jayaram V, Leonard MK, Travis KE, Gygi B, et al. Speech-specific tuning of neurons in human superior temporal gyrus. Cereb Cortex 2014;24:2679-93. doi:10.1093/cercor/bht127.

[62] Dykstra AR, Chan AM, Quinn BT, Zepeda R, Keller CJ, Cormier J, et al. Individualized localization and cortical surface-based registration of intracranial electrodes. NeuroImage 2012;59:3563-70. doi:10.1016/j.neuroimage.2011.11.046.

[63] Vitacco D, Brandeis D, Pascual-Marqui R, Martin E. Correspondence of event-related potential tomography and functional magnetic resonance imaging during language processing. Hum Brain Mapp 2002;17:4-12. doi:10.1002/hbm.10038.

[64] Fan J, Kolster R, Ghajar J, Suh M, Knight RT, Sarkar R, et al. Response Anticipation and Response Conflict: An Event-Related Potential and Functional Magnetic Resonance Imaging Study. J Neurosci 2007;27:2272-82. doi:10.1523/JNEUROSCI.3470-06.2007.

[65] Herzmann G, Jin M, Cordes D, Curran T. A within-subject ERP and fMRI investigation of orientation-specific recognition memory for pictures. Cogn Neurosci 2012;3:174-92. doi:10.1080/17588928.2012.669364.

[66] Oostenveld R, Fries P, Maris E, Schoffelen J-M. FieldTrip: open source software for advanced analysis of MEG, EEG, and invasive electrophysiological data. Comput Intell Neurosci 2010;2011:e156869. doi:10.1155/2011/156869.

[67] Franaszczuk PJ, Bergey GK. Application of the directed transfer function method to mesial and lateral onset temporal lobe seizures. Brain Topogr 1998;11:13-21.

[68] Kramer MA, Eden UT, Kolaczyk ED, Zepeda R, Eskandar EN, Cash SS. Coalescence and fragmentation of cortical networks during focal seizures. J Neurosci 2010;30:10076-85. doi:10.1523/JNEUROSCI.6309-09.2010.

[69] Martin S, Brunner P, Holdgraf C, Heinze H-J, Crone NE, Rieger J, et al. Decoding spectrotemporal features of overt and covert speech from the human cortex. Front Neuroengineering 2014;7:14. doi:10.3389/fneng.2014.00014.

[70] Murta T, Leal A, Garrido MI, Figueiredo P. Dynamic Causal Modelling of epileptic seizure propagation pathways: a combined EEG-fMRI study. NeuroImage 2012;62:1634-42. doi:10.1016/j.neuroimage.2012.05.053.

[71] Basu I, Kudela P, Korzeniewska A, Franaszczuk PJ, Anderson WS. A study of the dynamics of seizure propagation across micro domains in the vicinity of the seizure onset zone. J Neural Eng 2015;12:046016. doi:10.1088/1741-2560/12/4/046016.

[72] Wilke C, Lei Ding, Bin He. Estimation of time-varying connectivity patterns through the use of an adaptive directed transfer function. IEEE Trans Biomed Eng 2008;55:2557-64. doi:10.1109/TBME.2008.919885.

[73] Ding M, Mo J, Schroeder CE, Wen X. Analyzing coherent brain networks with Granger causality. 2011 Annu. Int. Conf. IEEE Eng. Med. Biol. Soc. EMBC, Boston, MA: IEEE; 2011, p. 5916-8. doi:10.1109/IEMBS.2011.6091463.

[74] Barnett L, Seth AK. The MVGC multivariate Granger causality toolbox: A new approach to Granger-causal inference. J Neurosci Methods 2014;223:50-68. doi:10.1016/j.jneumeth.2013.10.018.

[75] Smith AC, Brown EN. Estimating a state-space model from point process observations. Neural Comput 2003;15:965-91. doi:10.1162/089976603765202622.

[76] Smith AC, Frank LM, Wirth S, Yanike M, Hu D, Kubota Y, et al. Dynamic analysis of learning in behavioral experiments. J Neurosci 2004;24:447-61. doi:10.1523/JNEUROSCI.2908-03.2004. 
[77] Srinivasan L, Eden U, Willsky A, Brown E. A state-space analysis for reconstruction of goal-directed movements using neural signals. Neural Comput 2006;18:2465-94. doi:10.1162/neco.2006.18.10.2465.

[78] Prerau MJ, Smith AC, Eden UT, Yanike M, Suzuki WA, Brown EN. A mixed filter algorithm for cognitive state estimation from simultaneously recorded continuous and binary measures of performance. Biol Cybern 2008;99:1-14. doi:10.1007/s00422-0080227-z.

[79] Yousefi A, Paulk AC, Deckersbach T, Dougherty DD, Eskandar EN, Widge AS, et al. Cognitive state prediction using an EM algorithm applied to gamma distributed data. Eng. Med. Biol. Soc. EMBC 2015 37th Annu. Int. Conf. IEEE, IEEE; 2015, p. 7819-24.

[80] Afshar P, Khambhati A, Carlson D, Dani S, Lazarewicz M, Cong P, et al. A translational platform for prototyping closed-loop neuromodulation systems. Front Neural Circuits 2013;6:117. doi:10.3389/fncir.2012.00117.

[81] Stypulkowski PH, Stanslaski SR, Jensen RM, Denison TJ, Giftakis JE. Brain stimulation for epilepsy - local and remote modulation of network excitability. Brain Stimulat 2014;7:350-8. doi:10.1016/j.brs.2014.02.002.

[82] Bjune CK, Marinis TF, Brady JM, Moran J, Wheeler J, Sriram TS, et al. Package architecture and component design for an implanted neural stimulator with closed loop control. Eng. Med. Biol. Soc. EMBC 2015 37th Annu. Int. Conf. IEEE, IEEE; 2015, p. 7825-30.

[83] Wheeler JJ, Baldwin K, Kindle A, Guyon D, Nugent B, Segura C, et al. An implantable 64channel neural interface with reconfigurable recording and stimulation. Eng. Med. Biol. Soc. EMBC 2015 37th Annu. Int. Conf. IEEE, IEEE; 2015, p. 7837-40. 